\title{
ПРИМЕНЕНИЕ ГИПЕРСПЕКТРАЛЬНЫХ МЕТОДОВ В ГЕОЛОГИИ: СОСТОЯНИЕ И ПЕРСПЕКТИВЫ
}

\author{
В.А. Яценко ${ }^{1,2}$, Н.Д. Воронов ${ }^{2}$, В.В. Гниденко ${ }^{3}$, Н.В. Наливайчук ${ }^{3}$ \\ ${ }^{1}$ Научный фонд ученых и специалистов по молекулярной кибернетике и информатике, \\ просп. Акад. Глушкова, 40, корп. 4/1, Киев 03680, Украина, е-mail: vyatsenko@gmail.com \\ ${ }^{2}$ Институт космических исследований НАН Украины и ГКА Украины, \\ просп. Акад. Глушкова, 40, корп. 4/1, Киев 03680, Украина \\ ${ }^{3}$ Национальный технический университет Украины “Киевский политехнический институт”, \\ nросп. Победы, 37, Киев 03056, Украина, e-mail: nnv@scs.ntu-kpi.kiev.иа
}

\begin{abstract}
Описана информационная технология прогнозной оценки нефтегазоносности территории. Предложен метод опорных векторов для классификации векторов наиболее информативных признаков с учетом процедуры валидации. Разработан новый датчик для гравиметрических измерений, основанный на конкурентоспособном адаптивном криогенном чувствительном элементе. Получены оценки вероятности нефтегазоносности территорий по материалам дистанционных исследований. Комплексирование материалов дистанционных исследований с данными геолого-геофизических исследований повышает достоверность и эффективность результатов прогноза.
\end{abstract}

Ключевые слова: гиперспектральный метод, гиперон, гравиметр, земная поверхность, море, залежи нефти и газа, обнаружение, прогнозирование, SVM-метод.

Введение. Дистанционное зондирование Земли (ДЗ3) появилось в 1960-е годы, когда на первые космические аппараты ставили аппаратуру трассового типа - проекция области измерений на поверхность Земли представляла собой линию. В настоящее время уже развита и широко применяется аппаратура панорамного типа - сканеры, проекция области измерений на поверхность Земли которых представляет собой полосу. Повсеместное использование радиометров и гиперспектрометров при ДЗ3 позволило накопить огромный опыт и подготовить необходимую экспериментальную базу применения спектральных методов для обнаружения полезных ископаемых $[1,3]$.

В зависимости от необходимых исследований подбирается аппаратура с чувствительными элементами в нужном волновом диапазоне и необходимой точности. Однако пропускание атмосферы накладывает дополнительные ограничения. В большинстве случаев аппаратура спектрометров использует только часть интервала пропускания. Пропускание - величина, обратная поглощению атмосферы. Обе величины выражают в процентах.

Изучение свойств минералов и горных пород основано на минералогических и петрографических исследованиях. Известно, что более $92 \%$ земной коры по массе составляют силикаты - соли кремниевых кислот, поэтому их исследованию уделяется больше внимание. В видимом диапазоне минералы мало различимы, однако значительно больше различий наблюдается в ближнем инфракрасном диапазоне - такие группы минералов, как глины, карбонаты и сульфаты, имеют отчетливые линии поглощения, легко регистрируемые с помощью современных устройств ДЗ3.

Спектры горных пород отличаются меньшей контрастностью по сравнению со спектрами отдельных минералов. Вместе с тем для всех горных пород характерно увеличение коэффициента спектральной яркости (КСЯ) от видимого диапазона к ближнему инфракрасному. Для большинства горных пород наблюдается пологий максимум в области частот 2,0-2,7 мкм. Исследования показали что современная аппаратура ДЗЗ имеет достаточно высокую точность при использовании ее в геологии. Наибольшей проблемой остаются атмосферная коррекция и калибровка, что можно решить с помощью калибровочных полигонов. Отметим что существенные ограничения накладываются также освещенностью исследуемой территории, температурой поверхности и растительностью на месте.

Методика прогнозной оценки нефтегазоносности территории. Для создания количественных карт прогнозной оценки перспективности территории на поиски залежей нефти и газа предлагается методика оценки нефтегазоносности территории на основе интегрированного использования данных Д33 (гиперспектральных, спектральных, гравиметрических и др.). На всех этапах работ для систематизации, хранения и обработки информации широко используются ГИС-технологии.

Прогнозная оценка перспективности территории основывается на использовании связи залежей углеводородов (УВ) с разрывными структурами разного масштабного уровня, узлами 
пересечения разломов, кольцевыми структурами, антиклинальными складками. Учитывается проявление залежей УВ в спектральных яркостях растительного покрова [1].

Прогноз залежей проводится на основе учета всего комплекса признаков. Для оценки перспективности территории используют разные методы прогноза, основанные на SVM-методе. В результате получают оценку вероятности обнаружения объектов прогноза в каждой точке исследуемой территории, которая отвечает минимальной площади, распознаваемой на космических снимках. Достоверность результатов прогноза подтверждается численным моделированием, данными бурения на участках проведения работ.

Гравиметрические измерения на основе сверхпроводящего датчика нового типа. Современное состояние разработок в области гравитационных измерений в геологии не обеспечивает в полной мере исследование важных аспектов современных проблем ввиду отсутствия гравиметров с высокой чувствительностью. Ниже рассмотрен новый датчик для гравиметрических измерений, основанный на явлении магнитной левитации [4, 7, 19].

Оптокриогенный высокоточный гравиметр ориентирован на поиск полезных ископаемых, выявление очагов готовящихся землетрясений, проведение геофизических и астрофизических исследований в стационарных и полевых условиях [12-16, 18]. Гравиметр позволяет выявлять и исследовать нефтегазоносные линзы, рудные тела, алмазные трубки и другие полезные ископаемые, а также обнаруживать, выявлять вулканические камеры, тектонические разломы, плотностные неоднородности, границы геосфер и другие научно-исследовательские объекты. Прибор применим в стационарных и полевых условиях любой сложности, для морских и аэрокосмических исследований, при подготовке строительных и горнопроходческих работ. Действие прибора основано на явлении магнитной потенциальной ямы с автоматической стабилизацией положения равновесия пробного тела [8-11]. Следует заметить, что рынок гравиметров, пригодных для работы в полевых условиях, практически не заполнен. Лидером (после слияния с американской производителем “Лакоста-Ромберг”) является канадская фирма "Синтрекс".

Для решения задачи детализации гравитационного поля Земли используются измерения гравитационных градиентов по трем пространственным направлениям на основе трехосного гравитационного градиентометра, который может быть установлен на борту спутника, и определение орбиты спутника с помощью аппаратуры спутниковой навигации. Конечными результатами может быть разработка методики и алгоритмов, а также уточнение тонкой структуры грави- тационного поля Земли на основе математической обработки спутниковых градиентометрических измерений европейского спутника GOCE. Учеными разных стран проводятся детальные измерения параметров гравитационного поля с использованием разных типов гравиметров.

Гравиметр предназначен для измерений ускорения силы тяжести $g\left[\mathrm{M} / \mathrm{c}^{2}\right]$ при решении фундаментальных геофизических, геодезических и метрологических задач. В данное время точность наилучших стационарных наземных гравиметров составляет $10^{-8} \mathrm{~g}$, для гравиметров морского базирования $-10^{-7} \mathrm{~g}$, для авиационного $-10^{-6} \mathrm{~g}$. Большинство гравиметров, выпускаемых промышленностью, основано на свойствах растянутой пружины или упругих свойствах пружин, изготовленных из кварца или других специальных сплавов. Их точность недостаточна для решения указанных проблем. Поскольку погрешность гравиметров на основе традиционных принципов в целом исчерпана, многие разработчики на протяжении последних десятилетий стараются использовать нетрадиционные подходы к созданию суперточных гравиметров. Эти подходы можно сгруппировать по способу неконтактного подвешивания чувствительной массы гравиметра, использованию электрических или магнитных сил, методам измерения смещений чувствительной массы гравиметра (системы оптической регистрации, эффект Джозефсона как основа измерений и т. п.), а также по компьютерным методам обработки сигналов. Скачок в усовершенствовании гравиметров стал возможным благодаря финансированию разработок сверхпроводящих гравиметров. Базовая конструкция сверхпроводящего гравиметра остается неизменной на протяжении почти 30 лет. Свободное состояние (левитация) чувствительной массы этого гравиметра достигается на основе эффекта Браунбека-Мейснера.

Одна из актуальных задач механики свободного тела в магнитном поле - составление и анализ уравнений динамики свободного тела в поле идеально проводящих токовых контуров. Это связано с возможностью использования эффектов диамагнитного отталкивания и минимума магнитной потенциальной энергии, что позволяет стабилизировать равновесие свободного тела. При этом используется эффект минимума магнитной энергии и стойкости равновесия свободного идеально проводящего токового кольца, соосного в положении равновесия к неподвижному, идеально проводящему токовому кольцу. Выполнены исследования уравнения движения и устойчивости при наличии не одного, а двух неподвижных, идеально проводящих токовых колец, которые представляют собой консервативную потенциальную систему. Для составления уравнений динамики используется метод Лагранжа, разви- 
тый [10] относительно электромеханических преобразователей энергии. В качестве кинетической энергии выбрана энергия магнитного поля.

В настоящее время разрабатываются спутниковые гравиметры для получения оперативной информации о гравитационном поле Земли. Для реализации чувствительного элемента криогенного гравиметра используются сверхпроводящие наноматериалы, ориентированные на азотные температуры [13, 14]. Предложены также численные алгоритмы для оценки вариаций ускорения силы тяжести при решении геофизических задач [2].

В результате экспериментальных исследований общей работы по определению свойств рабочего макета гравиметра, динамических характеристик магнитного подвеса свободной пробной массы рабочего макета гравиметра (совместно с группой Института физики НАН Украины) доработан чувствительный элемент магнитного подвеса $[5,6,17]$.

На рисунке показана функциональная схема измерительно-вычислительной системы. Основные составные части этой системы: сверхпроводящий подвес, оптическая система регистрации информации и микропроцессорный вычислительный блок. В системе используется лазерный интерферометр для измерения малых смещений левитирующего пробного тела.

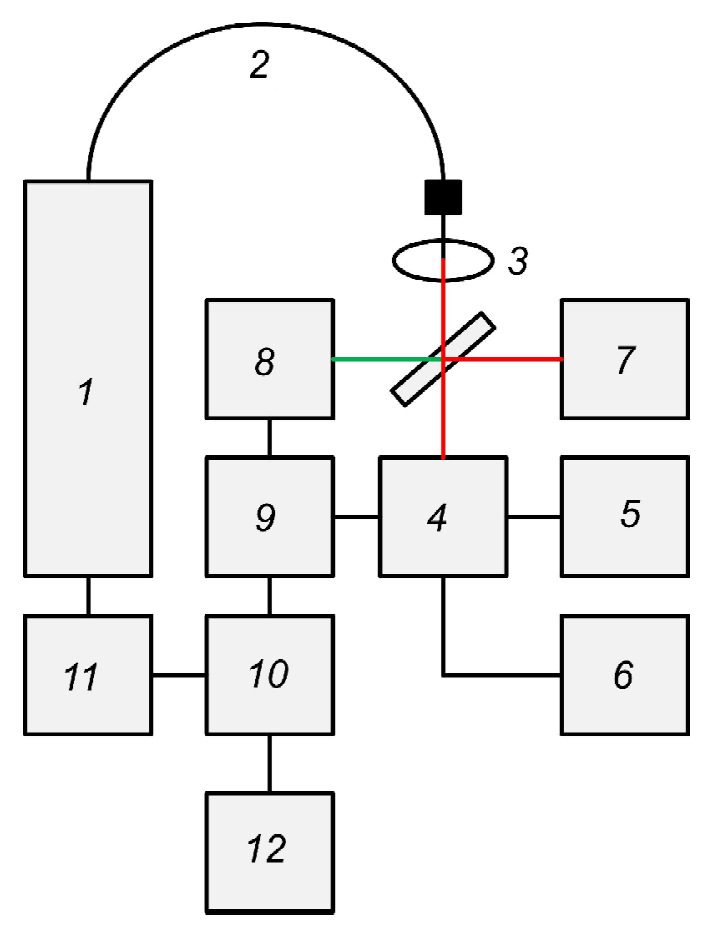

Функциональная схема измерительно-вычислительной системы: 1 - криостат со вставкой, на котором смонтирована оптическая головка; 2 - оптический кабель; 3 - фокусирующий объектив; 4 - головка с лазерным диодом; 5 - блок питания лазерного диода; 6 - блок стабилизации температуры; 7 - интерферометр Фарби-Перо ИФП-1; 8 - фотоприемник; 9 - синхронный детектор; 10 - микроконтроллер со встроенным ЦАП и АЦП; 11 - модулятор; 12 - блок клавиатуры и индикации
Обработка сигналов оптокриогенного гравиметра проводится в несколько этапов: на первом компенсируются шумы, влияющие на механическую часть прибора, втором - используется обратная динамическая модель датчика, на третьем - осуществляется адаптивная цифровая фильтрация. Сочетание таких принципов, как подвешивание свободной чувствительной массы, применение оптической регистрационной системы и новых средств обработки сигнала, обеспечивает новые динамические свойства прибора.

Разработана многомерная нелинейная математическая модель гравиметра, на основе которой получено билинейное, а затем и линейное приближение. Для оценки вектора состояний системы предложен робастный алгоритм гарантированного эллипсоидального оценивания множества достижимости управляемой линейной системы, подверженной влиянию ограниченной помехи с неизвестными статистическими характеристиками. Критерий точности - минимизация определителя матрицы эллипсоида.

Разработанный оптоволоконный криогенный гравиметр может быть использован при проведении фундаментальных исследований. Возможно его практическое применение в геодинамике, геологии, навигации, космических исследованиях, при изучении зарождения и развития критических локальных и глобальных состояний Земли, выявлении полезных ископаемых и решении других проблем. Первый шаг в реализации такой программы заключается в разработке наземного гравиметра на основе созданного нами оптокриогенного датчика. Он может быть использован совместно с сейсмометрами и магнитометрами при прогнозировании землетрясений, а также в сейсмометрии.

Применение данного прибора в микроспутнике ограничено отсутствием криогенного обеспечения его массогабаритными характеристиками. Обсуждение этой проблемы в КБ “Южное” и анализ современного состояния технологий показало, что для ее решения необходимо использовать наноразмерные структуры и материалы, что, в свою очередь, приведет к созданию датчика микромехатронных размеров. Определены материалы, которые могут быть использованы в разработке перспективных гравиметров и акселерометров. Реализация такой возможности позволит разработать приборы нового типа для микроспутников.

Прогнозная оценка нефтегазоносности территории. Прогнозная оценка территории основана на следующей последовательности работ.

1. Сбор и систематизация имеющихся данных (гиперспектральных, спектральных, гравиметрических и др.) для проведения прогнозных исследований, выделение наиболее информа- 
тивных признаков, обеспечивающих последующую компьютерную обработку $[1,3]$.

2. Анализ информативности собранных данных с использованием метода главных компонент для решения задач прогноза и формирование вектора поисковых признаков.

3. Адаптация разработанных программ прогноза для конкретных геолого-геофизических условий.

4. Расчеты прогнозной оценки перспективности территории на обнаружение залежей УВ по комплексу выбранных поисковых признаков с использованием специальных математических методов.

5. Анализ результатов прогнозной оценки перспективности территории на обнаружение залежей УВ с использованием экспертных оценок и подготовка рекомендаций на проведение дальнейших работ.

Работа с использованием метода распознавания образов состоит из трех этапов: первый формирование вектора наиболее информативных признаков объектов поиска в пространстве используемых поисковых признаков (структурных, спектральных и др.), второй - решение задачи распознавания образов с использованием SVMметода (SVM - support vector machines), третий сравнение всей изучаемой территории со сформированным “образом” объектов поиска. Прогнозной оценкой нефтегазоносности служит мера сходства участков территории с “образом” объекта прогноза.

Подход к обработке наиболее информативных признаков базируется на системном использовании нелинейных методов фильтрации, классификации и распознавания образов. Метод опорных векторов (SVM) - это набор похожих алгоритмов вида “обучение с учителем", которые используются для задач классификации и регрессионного анализа. Данный метод принадлежит к семейству линейных классификаторов. Он может также рассматриваться как специальный случай регуляризации, по А.Н. Тихонову. Особое свойство метода опорных векторов - беспрерывное уменьшение эмпирической ошибки классификации и увеличение зазора, в связи с чем метод также известен как метод классификатора с максимальным зазором.

Основная идея метода опорных векторов перевод исходных векторов в пространство более высокой размерности и поиск разделяющей гиперплоскости с максимальным зазором в этом пространстве. Две параллельные гиперплоскости строятся с обеих сторон гиперплоскости, разделяющей классы $k$. Разделяющая гиперплоскость максимизирует расстояние до двух соответствующих параллельных гиперплоскостей. Алгоритм работает в предположении, что, чем больше раз- ность или расстояние между параллельными гиперплоскостями, тем меньше средняя ошибка классификатора.

Найдена пара гиперплоскостей, которые дают максимальную ширину полосы, путем решения задачи оптимизации с ограничениями

$$
\min _{\mathbf{w}} \frac{1}{2}\|\mathbf{w}\|^{2}
$$

при условии

$$
z_{j^{\prime}}\left(\mathbf{x}_{j^{\prime}} \cdot \mathbf{w}+b\right)-1 \geq 0 \quad \forall j^{\prime}, j^{\prime}=1, \ldots, J^{\prime} .
$$

Один из подходов к классификации наиболее информативных признаков на $k$ классов, в зависимости от структуры района поиска, основан на методе опорных векторов. Для обучения алгоритма использованы эталонные структуры. Валидация модели классификации выполнялась с использованием знаний экспертов. Результаты классификации данных сравнивались с результатами классификации экспертами. Если результаты различались, предлагалось заново научить алгоритм классификации. Экспериментальные исследования показали высокую вероятность правильного распознавания предполагаемого района поиска.

\section{Выводы}

1. Описаны перспективные информационные технологии прогнозной оценки нефтегазоносности территории. Предложены методы математического моделирования и классификации для использования в геологии.

2. Предложено использовать метод опорных векторов для классификации векторов наиболее информативных признаков с учетом процедуры валидации. Методы исследования могут быть использованы в различных областях знаний, в том числе в экологии и сельском хозяйстве.

3. Прогнозные модельные исследования позволяют получить оценки вероятности нефтегазоносности территорий по материалам дистанционных исследований с учетом геологических и природных условий.

4. Комплексирование материалов дистанционных исследований с данными геолого-геофизических исследований повышает достоверность и эффективность результатов прогноза. Совместное применение системы векторного сканирования, гравитационного моделирования и корреляционного анализа позволяет построить геологическую модель, адекватную априорной геологической информации и наблюденному полю.

5. Предложенная методика прогноза нефтегазоносности позволяет: а) проводить оценку перспективности территории на нефть и газ 
независимо от времени года и природных условий; б) выдавать рекомендации по постановке детальных поисковых работ; в) оперативно проводить прогнозные исследования на любой территории в требуемом масштабе; г) выделять локальные участки с максимально возможной вероятностью обнаружения залежей нефти и газа, таким образом сокращая финансовые затраты и сроки выполнения поисково-разведочных работ.

1. Архипов А.И. Методология и опыт поиска залежей углеводородов на суше с использованием аэрокосмической информации /А.И. Архипов, 3.М. Товстюк, О.И. Левчик, Т.А. Архипова, Т.В. Осканьян, В.И. Лялько, М.А. Попов // Науки про Землю та космос суспільству: праці Першої наук. конф., Київ, 2527 черв. 2007. - К., 2007.- С. 55-60.

2. Гніденко B. Нейромережеве оцінювання слабких впливів на кероване левітуюче пробне тіло /В. Гніденко, М. Наливайчук, В. Яценко // Наук. праці Нац. ун-ту харч. технологій. - 2013.- № 48. - С. 44-48.

3. Горный В.И. Обзор достижений последнего десятилетия в области применения спутниковых методов дистанционного зондирования при геологических и геофизических исследованиях / В.И. Горный, А.А. Тронин // Современные проблемы дистанционного зондирования Земли и космоса. $-2012 .-$ Т. 9 , № 5. - С. 116-132.

4. Козорез В.В. Динамические системы свободных магнитно-взаимодействующих тел / В.В. Козорез. Киев: Наук. думка, 1981. - 140 с.

5. Наливайчук М. Вимірювально-обчислювальна система для отримання оперативної інформації щодо гравітаційного поля /М. Наливайчук., В. Яценко, В. Гніденко // Комп’ютерно-інтегровані технології: освіта, наука, виробництво. - Луцьк: Вид-во Луцьк. нац. техн. ун-ту, 2013. - № 12. - С. 167-173.

6. Наливайчук Н. Аппаратно-программное обеспечение адаптивного оптокриогенного гравиметра на основе наноструктур / Н. Наливайчук, В. Яценко // 13-та укр. конф. $з$ косм. досліджень: Зб. тез. - К.: Кафедра, 2013. - C. 139.

7. Яценко В.О. Моделювання та оптимізація адаптивного кріогенного гравіметра / В.О. Яценко, М.В. Наливайчук // 19-та Міжнарод. конф. $з$ автомат. керування, Київ, 26-28 вер., 2012. - К., Україна, 2012. - С. 344.
8. Goodkind J.M. The superconducting gravimeter / J.M. Goodkind // Rev. Sci. Instrum. - 1999. - V. 70, No. 11. - P. 4131-4152.

9. Goodkind J.M. Superconductivity applied to gravimetry / J.M. Goodkind, R.J. Warburton // IEEE Transactions on Magnetics. - 1975. - V. 11, No. 2. - P. 708-711.

10. Kozoriz $V$. Novel Magnetic Levitation and Propulsion Phenomena / V. Kozoriz. - Zaporizhya, 1999. - 271 p.

11. Moon F.C. Superconducting Levitation: Application to bearings and magnetic transportation. - New York: John Willey \& Sons, 1994. -295 p.

12. Prothero W.A. A superconducting gravimeter / W.A. Prothero, J. M. Goodkind // Rev. Sci. Instr. 1968. - V. 39.- P. 1257- 1261.

13. Yatsenko V. Cryogenic-Optical Gravimeter: Principles, Methods and Applications / V. Yatsenko, M. Nalyvaichuk // Kharkov Univer. Vestnik. Ser. Radiophysics and Electronics. - 2011.- P. 107-113.

14. Yatsenko V. Global optimization of cryogenic-optical sensor, in Sensors, Systems, and Next-Generation Satellites / V. Yatsenko, P. Pardallos; eds K.W.H. Fujisada, J. Lirie. Proc. SPIE 4550. - 2001. - P. $433-441$.

15. Yatsenko $V$. Modeling and Optimization of CryogenicOptical Gravimeters / V. Yatsenko, M. Nalyvaichuk // Inter. J. information theories and applications. - 2012. V. 19, No. 3.- P. 232-240.

16. Yatsenko $V$. Modeling of Superconducting Gravimeter for Futute Space Missions: Optimization. Control, and Estimation / V. Yatsenko, M. Nalyvaichuk, V. Gnidenko, O. Kochkoda // 2nd Inter. conf. on memory of corresponding member of Nat. Acad. Sci. of Ukraine V.S. Melnik, Kyiv, 4-6 Apr., 2012. - Kyiv, Ukraine 2012. - P.110.

17. Yatsenko $V$. Opto-cryogenic sensitive element with ultrasensitive laser interferometer and microprocessor controller / V. Yatsenko, N. Nalivaichuk // IEEE Inter. conf. on Advanced Optoelectronics and Lasers, CAOL, : abstr. - Kharkov, UIRE, 2013. - P. 39.

18. Yatsenko V.A. On the possibility of application of "Magnetic Potential Well" effect for creation of a graviinertial cryogenic device / V.A. Yatsenko, E.I. Kryuchkov // J. Automation and Information Sci. -2003 - V. 34. P. 106-119.

19. Yatsenko V.A. Mathematical modeling and optimization of controlled superconducting sensors with magnetic levitation / V.A. Yatsenko, N.V. Nalivaichuk // Моделювання та дослідження стійкості динамічних систем: Зб. наук. праць (міжнар. конф. DSMSI-2011). - K., 2011. - C. 146. 


\title{
ЗАСТОСУВАННЯ ГІПЕРСПЕКТРАЛЬНИХ МЕТОДІВ У ГЕОЛОГІЇ: СТАН І ПЕРСПЕКТИВИ
}

\author{
В.О. Яценко ${ }^{1,2}$, М.Д. Воронов ${ }^{2}$ В.В. Гніденко ${ }^{3}$, М.В. Наливайчук ${ }^{3}$ \\ ${ }^{1}$ Науковий фонд вчених та спеціалістів з молекулярної кібернетики і інформатики, \\ nросп. Акад. Глушкова, 40, корп. 4/1, Київ 03680, Україна, e-mail: vyatsenko@gmail.com \\ ${ }^{2}$ Інститут космічних досліджень НАН України та ГКА України, \\ просп. Акад. Глушкова, 40, корп. 4/1, Київ 03680, Україна \\ ${ }^{3}$ Національний технічний університет Украӥни “Київський політехнічний інститут", \\ nросn. Перемоги, 37, Київ 03056, Україна, e-mail: nnv@scs.ntu-kpi.kiev.ua
}

\begin{abstract}
Описано інформаційну технологію прогнозної оцінки нафтогазоносності території. Запропоновано метод опорних векторів для класифікації векторів найінформативніших ознак з урахуванням процедури валідації. Розроблено новий датчик для гравіметричних вимірювань, що грунтується на використанні конкурентоспроможного адаптивного кріогенного чутливого елемента. Отримано оцінки ймовірності нафтогазоносності територій за матеріалами дистанційних досліджень. Комплексування матеріалів дистанційних досліджень 3 даними геологогеофізичних досліджень підвищує вірогідність і ефективність результатів прогнозу.
\end{abstract}

Ключові слова: гіперспектральний метод, гіперон, гравіметр, земна поверхня, море, поклади нафти і газу, виявлення, прогнозування, SVM-метод.

\section{HYPERSPECTRAL METHODS IN GEOLOGY: CURRENT STATE AND PROSPECTS}

\author{
V.A. Yatsenkol,2, N.D. Voronov', V.V. Gnidenko ${ }^{3}$, N.V. Nalivaychuk ${ }^{3}$ \\ ${ }^{1}$ Scientific Foundation of Scientists and Spesialists on Molecular Cybernetics and Informatics, \\ Academica Glushkova Ave., 40, bild. 4/1, Kyiv 03680,Ukraine, e-mail: vyatsenko@gmail.com \\ ${ }^{2}$ Space Research Institute of NASU and SSAU, Academica Glushkova Ave., 40, bild. 4/1, Kyiv 03680, Ukraine \\ ${ }^{3}$ National Technical University of Ukraine "Kyiv Polytechnic Institute”, Peremogy Ave., 37, Kyiv 03056, Ukraine, \\ e-mail:nnv@scs.ntu-kpi.kiev.ua
}

Purpose. Remote sensing (RS) began in the early 60s with the development of image processing of satellite imagery. Wide use of radiometers and hyperspectrometers in RS led to the accumulation of huge volume of experimental data that can now be used for remote detection of minerals. Imaging spectrometry data or hyperspectral imagery acquired by airborne systems have been used in geologic since the early 1980 's and represent a mature technology. The solar spectral range $0,4-$ $2,5 \mu \mathrm{m}$ provides abundant information about hydroxyl-bearing minerals, sulfates and carbonates common to many geologic units and hydrothermal alteration assemblages. The purpose of this paper is to show the feasibility of hybrid information technology and new sensors for identification of oil and gas.

Design/methodology/approach. We propose to combine hyperspectral, spectral and gravimetric data for oil forecasting using most informative parameters and the SVM-method. Behind the method is the idea of intelligent analysis based on models. Guided by this methodology, we demonstrate some possibilities involving four types of data such as hyperspectral, gravimetric, seismic, and geological data. Our method is also based on Spectral Angle Mapper (SAM) as a tool for matching the separated and members with pure spectra from databases. The analysis of SAM is provided in three spectral regions: VIS, IR and VIS+IR combined. Three kinds of available influences can be analysed: additive noise, constant offset, and slant offset.

Findings. This paper presents an overview of support vector machines (SVM) as one of the most promising intelligent techniques for data analysis, as theoretical approaches and sophisticated applications developed for various research areas and problem domains. It is an attempt to provide a survey of the applications of SVM for oil and gas exploration. The applications of SVM have been grouped and summarized in the different areas of the exploration phase, which can be used as a guide to assess the effectiveness of SVM as against other data mining algorithms. The study introduces an image specific algorithm for oil identification and discusses the implications for geology. Based on the hydrocarbon infiltration theory, gravimetric data, the analysis of crude oil in soil, spectral data of crude oil in sea water, and Hyperion hyperspectral remote sensing images were used to develop the synergetic approach to oil-gas exploration.

Practical value/implications. Our methodology proves to be practical for thorough data analysis in the exploration and production of oil and gas. The results indicate that the area of the oil-gas reservoir could be delimited in two ways: a) directly, by the absorption bands near $1730 \mathrm{~nm}$ in Hyperion image; b) indirectly, by using Linear Spectral Unmixing (LSU) and Spectral Angle Matching (SAM) of alteration mineral (e.g. kaolinite, illite). In addition, combined with the optimal bands in the region of visible/near-infrared, SAM can be used to extract the thin oil slick of microseepage.

Keywords: hyper spectral, hyperion, gravimeter, land, sea, oil-gas reservoir, detection, forecasting, SVM-method, mapper. 


\section{References}

1. Arhipov A.I., Tovstjuk Z.M., Levchik O.I., Arhipova Ljalko V I., Popov M.A. Metodologija i opyt poiska zalezhej uglevodorodov na sushe sispol'zovaniem ajerokosmicheskoj informacii [Methodology and experience finding hydrocarbon deposits on land using aerospace information]. Nauky pro zemlyu ta kosmos suspilstvu. Pratsi Pershoyi naukovoyi konferentsiyi [Earth science and space community. Proceedings of the First Conference, 25-27 chervnya 2007 r.]. Kyiv, 2007, pp. 55-60.

2. Hnidenko V., Nalyvaychuk M., Yatsenko V. Neiromerezheve otsiniuvannia slabkykh vplyviv na kerovane levituiuche probne tilo [Neural network estimation of weak effects on controlled levitation test subject]. Naukovi pratsi Natsionalnoho universytetu kharchovykh tekhnolohii [Proceedings of National University of food tehnolohiy], 2013, no. 48, pp. 44-48.

3. Gorny V. I., Tronin A. A. Obzor dostizhenij poslednego desjatiletija v oblasti primenenija sputnikovih metodov distancionnogo zondirovanija pri geologicheskih i geofizicheskih issledovanijah [Overview of the achievements of the last decade in the use of satellite remote sensing methods in geological and geophysical studies]. Sovremennye problemy distancionnogo zondirovanija Zemli i kosmosa [Modern Problems of Remote Sensing and Space], 2012, vol. 9, no. 5, pp. 116-132.

4. Kozorez V.V. Dinamicheskie sistemy svobodnyh magnitno vzaimodejstvujushhih tel [Dynamical systems magnetically interacting free subject]. Kyiv, Naukova dumka, 1981, 140 p.

5. Nalyvaychuk M., Yatsenko V., Hnidenko V. Vymiriuvalno-obchysliuvalna systema dlia otrymannia operatyvnoi informatsii shchodo hravitatsiinoho polia [Measurement and computer system to obtain timely information on the gravitational field]. Kompiuterno-intehrovani tekhnolohii: osvita, nauka, vyrobnytstvo [Integrated Computer: education, science, vyrobnytstvo]. Luck, Publisher Lutsk National Technical University, 2013, no. 12, pp. 167-173.

6. Nalivajchuk N., Yatsenko V. Apparatno programmnoe obespechenie adaptivnogo opto-kriogennogo gravimetra na osnove nanostruktur [Hardware software adaptive opto-cryogenic gravimeter based on nanostructures] Zbirnyk tez 13-yi ukrainskoi konferentsii z kosmichnykh doslidzhen. [Abstracts of the 13th Ukrainian Conference on Space doslidzhen]. Kyiv, Kafedra, 2013, 139 p.

7. Yatsenko V.O., Nalyvaychuk M.V. Modeliuvannia ta optymizatsiia adaptyvnoho kriohennoho hravimetra [Modeling and optimization of adaptive cryogenic gravimeters]. 19 Mizhnarodna konferentsiya z avtomatychnoho keruvannya, Kyiv, 26-28 veresnya 2012 [19th International Conference on Automatic Control, Kyiv 26-28 September 2012]. Kyiv, Ukraine, 2012, $344 \mathrm{p}$.

8. Goodkind J.M. The superconducting gravimeter. Rev. Sci. Instr., 1999, vol. 70, no. 11, pp. 4131-4152.

9. Goodkind J.M., Warburton R.J. Superconductivity applied to gravimetry. IEEE Transactions on Magnetics, 1975, vol. 11, no. 2, pp. 708-711.

10. Kozoriz V. Novel Magnetic Levitation and Propulsion Phenomena. Zaporizhya, 1999, 271 p.

11. Moon F.C. Superconducting Levitation: Application to bearings and magnetic transportation. New York, John Willey \& Sons, 1994, $295 \mathrm{p}$.

12. Prothero W.A., Goodkind. J. M. A superconducting gravimeter. Rev. Sci. Instr,1968, vol. 39, , no. 9, pp. $1257-1261$.

13. Yatsenko V., Nalyvaichuk M. Cryogenic-Optical Gravimeter: Principles, Methods and Applications. Kharkov University Vestnik, Ser. Radiophysics and Electronics, 2011, pp. 107-113.

14. Yatsenko V., Pardallos P. Global optimization of cryogenic-optical sensor, in Sensors, Systems, and Next-Generation Satellites. Eds K. W.H. Fujisada, J. Lirie. Proc. SPIE 4550, 2001, pp. 433-441.

15. Yatsenko V., Nalyvaichuk M. Modeling and Optimization of Cryogenic-Optical Gravimeters. International Journal information theories \& applications, 2012, vol. 19, no. 3, pp. 232-240.

16. Yatsenko V., Nalyvaichuk M., Gnidenko V., Kochkodan O. Modeling of Superconducting Gravimeter for Futute Space Missions: Optimization. Control, and Estimation. 2nd International Conference on memory of corresponding member of National Academy of Science of Ukraine V.S. Melnik, 4-6 April, 2012, Kyiv, Ukraine, 110 p.

17. Yatsenko V., Nalivaichuk N. Opto-cryogenic sensitive element with ultrasensitive laser interferometer and microprocessor controller. Abstracts. IEEE International Conference on Advanced Optoelectronics and Lasers, CAOL. Kharkov, UIRE, 2013, $39 \mathrm{p}$.

18. Yatsenko V.A., Kryuchkov E.I. On the possibility of application of "Magnetic Potential Well" effect for creation of a graviinertial cryogenic device. Journal of Automation and Information Science, 2003, vol. 34, pp. 106-119.

19. Yatsenko V.A., Nalivaichuk N.V. Mathematical modeling and optimization of controlled superconducting sensors with magnetic levitation. Modeliuvannia ta doslidzhennia stiikosti dynamichnykh system: Zbirnyk naukovykh prats mizhnarodna konferentsii DSMSI-2011 [The modeling and study of the stability of dynamical systems: Scientific Papers of the International Conference DSMSI-2011]. Kyiv, 2011, 146 p. 